\title{
Soret Effect due to Natural Convection between Heated Vertical Plates with Heat Generation in a Horizontal Small Magnetic Field
}

\author{
Bishwaram Sharma \\ Department of Mathematics, \\ Dibrugarh University, Dibrugarh- \\ 786004, Assam, India
}

\author{
Ram Niroj Singh \\ Marwari Hindi High School, \\ Jalukpara, Dibrugarh-786001, \\ Assam, India
}

\author{
Rupam Kr. Gogoi \\ Sibsagar College, Joysagar, \\ Joysagar-785665, \\ Assam, India
}

\begin{abstract}
The effect of a small uniform applied horizontal magnetic field on separation of a binary mixture for the case of the fully developed natural convection of a fluid between two heated vertical walls in presence of heat generation has been studied analytically. Neglecting the induced electric field the equations governing the motion, temperature and concentration are solved by series expansion in terms of dimensionless parameter measuring buoyancy force. The solution obtained for concentration distribution is plotted against the width of the channel. It is found that the dimensionless number measuring the buoyancy force, the Hartmann number measuring the magnetic force, the thermal diffusion number and the heat generation parameter affects the species separation of rarer and lighter component significantly.
\end{abstract}

\section{Keywords}

Binary mixture, incompressible fluids, natural convection, thermal diffusion, magnetic field, heat generation.

\section{INTRODUCTION}

The study of heat generation or absorption in moving fluids is important in problems dealing with chemical reactions and those concerned with dissociating fluids. Vajravelu and Hadjinicolaou[1] studied heat transfer characteristics in the laminar boundary layer of a viscous fluid over a stretching sheet with viscous dissipation or fractional heating and internal heat generation. In that study they considered that the volumetric rate of heat generation, $q^{\prime \prime \prime}\left[w . \mathrm{m}^{3}\right]$ should be $q^{\prime \prime \prime}=Q_{0}\left(T-T_{0}\right)$ for $T>T_{0}$ and equal to zero for $T<T_{0}$, where $Q_{0}$ is the heat generation/absorption constant. The above relation is valid as an approximation of the state of some exothermic process and having $T_{0}$ as the ambient temperature. When the inlet temperature is not less than $T_{0}$ they used $q^{\prime \prime \prime}=Q_{0}\left(T-T_{0}\right)$. The effect of conjugate conduction-natural convection heat transfer along a vertical plate with non-uniform heat generation was studied by Mendez and Trevino [2].

The general motivation, background, governing equations and boundary conditions to this problem area have been elaborated in [3] and avoiding the repetition in the interest of brevity without compromising the intelligibility of the present work. The current result may be regarded as a step in the direction of bringing the results of [4] closer towards eventual application to the study of basic fluid dynamics processes in the separation of binary mixture
In many cases the fluid mixture is found to be electrically conducting and so to study the effect of a magnetic field on separation, it is considered that a binary mixture of incompressible viscous thermally and electrically conducting fluids sheared between two parallel vertical plates in presence of a constant uniform horizontal magnetic field and heat generation. Using the expressions for the velocity distribution and temperature distribution as derived by Osterle and Young [5], it is investigated that the effect of magnetic field on the concentration distribution of the rarer component of a binary fluid mixture in presence of heat generation.

\section{FORMULATION OF THE PROBLEM}

Considering here the steady flow of a binary mixture of thermally and electrically conducting viscous incompressible fluids of very small electrical conductivity is sheared between two infinitely wide vertical plates at $\mathrm{y}=-\mathrm{d}$ and $\mathrm{y}=\mathrm{d}$ separated by a distance $2 \mathrm{~d}$.It is considered that the flow is symmetric about $\mathrm{x}$-axis which is along the channel and the $\mathrm{y}$ axis is in the horizontal direction. The plates are maintained at uniform temperature $\mathrm{T}_{1}$ which exceeds the ambient temperature $T_{0}\left(T_{0}<T_{1}\right)$. A horizontal magnetic field of uniform strength $\mathrm{B}_{0}$ is applied perpendicular to the plates. The flow of the fluid due to buoyancy force is in the direction parallel to the plates and is of magnitude u.The induced magnetic field is of the order of the product of magnetic Reynolds number and imposed magnetic field. As the flow discussed here is the case fully developed natural convection of a fluid with very small electrical conductivity, therefore, it is the case of low magnetic Reynolds number and hence the induced magnetic field due to the weak applied magnetic field may be neglected.

In fully developed flow the pressure distribution must be hydrostatic, hence

$$
\frac{\partial \mathrm{p}}{\partial \mathrm{x}}=-\rho_{0} \mathrm{~g}
$$

Where $\rho_{0}$ is the fluid density at ambient temperature and $\mathrm{g}$ is the acceleration due to gravity. In this case, the density $\rho$ varies slightly from point to point because of the variation in temperature $\mathrm{T}$ and can be expressed as

$$
\rho=\rho_{0}\left[1-\beta\left(T-T_{0}\right)\right],
$$


which is well known Boussinesq approximation, where

$$
\beta=-\frac{1}{\rho}\left(\frac{\partial \rho}{\partial \mathrm{T}}\right)_{\rho}
$$

is the expansivity of the fluid.

For the above assumption the governing equations for motion, temperature and species conservation for the steady flow of a binary mixture of incompressible thermally and electrically conducting viscous fluids sheared between two electrically non-conducting vertical parallel plates in presence of a uniform horizontal magnetic field and heat generation becomes

$v \frac{\partial^{2} \mathrm{u}}{\partial \mathrm{y}^{2}}+\beta g\left(\mathrm{~T}-\mathrm{T}_{0}\right)-\frac{\sigma \mathrm{uB}_{0}^{2}}{\rho}=0$,

$\kappa \frac{\partial^{2} \mathrm{~T}}{\partial \mathrm{y}^{2}}+\rho v\left(\frac{\partial \mathrm{u}}{\partial \mathrm{y}}\right)^{2}+\sigma \mathrm{B}_{0}^{2} \mathrm{u}^{2}+\mathrm{Q}_{0}\left(\mathrm{~T}-\mathrm{T}_{0}\right)=0$

and $\frac{\partial}{\partial y}\left(\frac{\partial \mathrm{C}_{1}}{\partial \mathrm{y}}+\mathrm{s}_{\mathrm{T}} \mathrm{c}_{1} \frac{\partial \mathrm{T}}{\partial \mathrm{y}}\right)=0$,

where $v=\frac{\mu}{\rho}$ is the coefficient of kinematic viscosity.

The equation of continuity suggests that

$$
\mathrm{u}=\mathrm{u}(\mathrm{y})
$$

By using (7) and the following assumptions

$\mathrm{U}=\frac{v \mathrm{u}}{\mathrm{g} \beta \mathrm{d}^{2}\left(\mathrm{~T}_{1}-\mathrm{T}_{0}\right)}, \quad \theta=\frac{\mathrm{T}-\mathrm{T}_{0}}{\mathrm{~T}_{1}-\mathrm{T}_{0}}, \quad c_{1}=\mathrm{c}_{0} \mathrm{c}, \mathrm{Q}=\frac{\mathrm{Q}_{0} \mathrm{~d}^{2}}{\mathrm{\kappa}}$ and

$\mathrm{Y}=\frac{\mathrm{y}}{\mathrm{d}}(8)$

in equations (4), (5) and (6) which can be placed in dimensionless form, yielding

$\frac{d^{2} U}{d Y^{2}}+\theta-M^{2} U=0$

$\frac{\mathrm{d}^{2} \theta}{d \mathrm{Y}^{2}}++\mathrm{N}\left(\frac{\mathrm{dU}}{\mathrm{dY}}\right)^{2}+\mathrm{M}^{2} \mathrm{NU} \mathrm{U}^{2}+\mathrm{Q} \theta=0$

$\frac{d}{d c}\left(\frac{d c}{d Y}+T_{d} c \frac{d \theta}{d Y}\right)=0$

The boundary conditions on velocity, temperature and concentration in terms of dimensionless quantities become

$\mathrm{U}=0, \theta=1, \mathrm{c}=1$ at $\mathrm{Y}=1$

$\operatorname{and} \frac{d U}{d Y}=0, \frac{d \theta}{d Y}=0, \frac{d c}{d Y}=0 \quad$ at $Y=0$.

where $\mathrm{M}^{2}=\frac{\sigma \mathrm{B}_{0}^{2} \mathrm{~d}^{2}}{\rho v}$ is the Hartmann number measuring the magnetic force, $\mathrm{N}=\frac{\rho^{2} \beta^{2} \mathrm{~d}^{4}\left(\mathrm{~T}_{1}-\mathrm{T}_{0}\right)}{\nu \kappa}$ is dimensionless number measuring the buoyancy force and $t_{d}=S_{T}\left(T_{1}-T_{0}\right)$ is the thermal diffusion number.

\section{SOLUTION OF THE PROBLEM}

The solutions of the equations (9) and (10) under the boundary conditions (12) and (13) have been obtained by Osterle and Young[5] by perturbing the velocity and temperature as

$\mathrm{U}=\mathrm{U}_{0}+\phi \mathrm{Nand} \theta=\theta_{0}+\varepsilon \mathrm{N}$

where

$\mathrm{U}_{0}=\frac{1}{\mathrm{M}^{2}+\mathrm{Q}}\left[\frac{\cos \sqrt{\mathrm{Q}} \mathrm{Y}}{\cos \sqrt{\mathrm{Q}}}-\frac{\operatorname{coshMY}}{\cos \mathrm{M}}\right]$,

$\theta_{0}=\frac{\cos \sqrt{\mathrm{Q}} \mathrm{Y}}{\cos \sqrt{\mathrm{Q}}}$

and

$$
\begin{aligned}
& \varepsilon=\frac{\mathrm{M}^{2}}{2 \mathrm{Q}\left(\mathrm{M}^{2}+\mathrm{Q}\right)^{2} \cos ^{2} \sqrt{\mathrm{Q}}}\left(\frac{\cos \sqrt{\mathrm{Q}} \mathrm{Y}}{\cos \sqrt{\mathrm{Q}}}-1\right)- \\
& \frac{\mathrm{M}^{2}}{6 \mathrm{Q}\left(\mathrm{M}^{2}+\mathrm{Q}\right)^{2} \cos ^{2} \sqrt{\mathrm{Q}}}\left(\frac{\cos \sqrt{\mathrm{Q}} \mathrm{Y}}{\cos \sqrt{\mathrm{Q}}} \cos 2 \sqrt{\mathrm{Q}}-\cos 2 \sqrt{\mathrm{Q} Y}\right)+ \\
& \frac{M^{2}}{\left(M^{2}+Q\right)^{2}\left(4 M^{2}+Q\right) \cosh ^{2} M}\left(\frac{\cos \sqrt{Q} Y}{\cos \sqrt{Q}} \cosh 2 M-\cosh 2 M Y\right)- \\
& \frac{2 \mathrm{M} \sqrt{\mathrm{Q}}}{\left(\mathrm{M}^{2}+\mathrm{Q}\right)^{2}\left(\mathrm{M}^{2}+4 \mathrm{Q}\right) \operatorname{coshM} \cos \sqrt{\mathrm{Q}}}\left(\frac{\cos \sqrt{\mathrm{Q}} \mathrm{Y}}{\cos \sqrt{\mathrm{Q}}} \sin \sqrt{\mathrm{Q}} \sinh \mathrm{M}-\right. \\
& \sin \sqrt{\mathrm{Q}} \mathrm{Y} \sinh \mathrm{MY})-\frac{2 \mathrm{M}^{2} \cos \sqrt{\mathrm{Q}} \mathrm{Y}}{\left(\mathrm{M}^{2}+\mathrm{Q}\right)^{2}\left(\mathrm{M}^{2}+4 \mathrm{Q}\right) \cosh \mathrm{Mcos} \sqrt{\mathrm{Q}}}(\cosh \mathrm{M}- \\
& \cosh M Y)+\frac{1}{2\left(M^{2}+Q\right)^{2} \cos ^{2} \sqrt{Q}}\left(\frac{\cos \sqrt{Q} Y}{\cos \sqrt{Q}}-1\right)+ \\
& \frac{1}{6\left(M^{2}+Q\right)^{2} \cos ^{2} \sqrt{Q}}\left(\frac{\cos \sqrt{Q} Y}{\cos \sqrt{Q}} \cos 2 \sqrt{Q}-\cos 2 \sqrt{Q} Y\right)- \\
& \frac{4 \mathrm{Q} \cos \sqrt{\mathrm{Q} Y}}{\left(\mathrm{M}^{2}+\mathrm{Q}\right)^{2}\left(\mathrm{M}^{2}+4 \mathrm{Q}\right) \cosh \mathrm{Mcos} \sqrt{\mathrm{Q}}}(\cosh \mathrm{M}-\cosh \mathrm{MY}) .
\end{aligned}
$$

Using above expressions for $U$ and $\theta$, the equation (11) is solved under the boundary conditions (12) and (13) and obtained

$$
c=e^{t_{d}(1-\theta)}
$$

\section{CONCLUSION}

In absence of magnetic field i.e. for $\mathrm{M}=0$, the concentration for the rarer component of the binary mixture can't be obtained directly from equation (18) because $\mathrm{M}$ appears as a factor in the denominator for the expression of $\theta$. So by putting $\mathrm{M}=0$ in equation (9), (10),(11) and solving under boundary conditions (12) and (13), obtained

$C=\mathrm{e}^{\mathrm{t}_{\mathrm{d}}\left(1-\frac{\cos \sqrt{\mathrm{Q} Y}}{\cos \sqrt{\mathrm{Q}}}-\mathrm{N} \varepsilon_{0}\right)}$,

where

$\varepsilon_{0}=\frac{1}{2 Q^{2} \cos ^{2} \sqrt{\mathrm{Q}}}\left(\frac{\cos \sqrt{\mathrm{Q} Y}}{\cos \sqrt{\mathrm{Q}}}-1\right)+\frac{1}{6 Q^{2} \cos ^{2} \sqrt{\mathrm{Q}}}\left(\cos 2 \sqrt{\mathrm{Q}} \frac{\cos \sqrt{\mathrm{Q} Y}}{\cos \sqrt{\mathrm{Q}}}-\right.$ $\cos 2 \sqrt{\mathrm{Q}} Y)$ 
Putting $t_{d}=0$ in (18) and (19) of concentration distribution for the rarer and the lighter component of binary fluid mixtureand get $c(Y)=1$ for all values of $Y$. From this it can be concluded that the separation of species ceases to take place if the effect of temperature gradient is neglected

Fig. 1, Fig. 2and Fig. 3 reveal that the rate of species separation decreases with the increase of the values of Q, N and $t_{d}$. This suggests that the rate of separation of species can be enhanced by decreasing the heat generation parameter and the temperature difference between the plates and the surrounding. Fig. 4 reveals that the rate of species separation enhances by the increase of magnetic field.

From the above discussion it can be concluded that the process of separation of the species of the binary mixture can be enhanced by decreasing the heat generation parameter, the thermal diffusion number, the dimensionless number measuring buoyancy force and by increasing the intensity of the applied magnetic field. Our conclusion are found to be in goodagreement with the results obtained by the researchers [6]-[26].

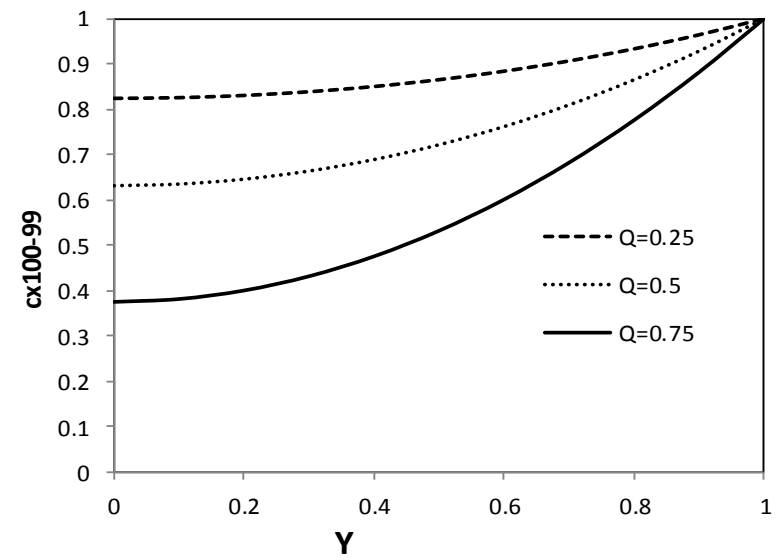

Fig.1. Graph of concentration function against the normalized distance for different values of $Q$ taking $t_{d}=0.01, M=0.5$ and $N=0.3$

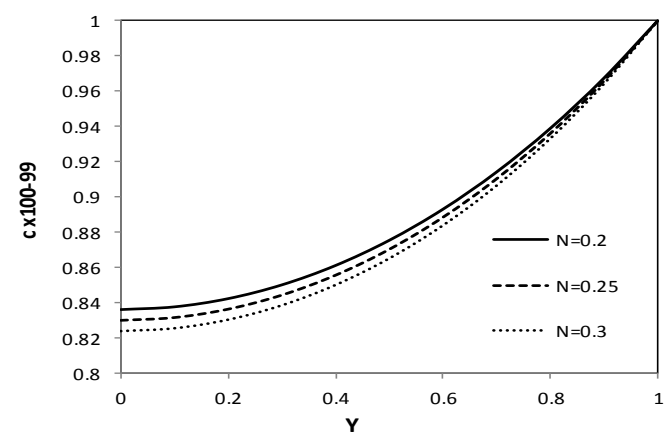

Fig.2. Graph of concentration distribution against the normalized distance for different values $N$ taking $t_{d}=0.01$, $M=0.5$ and $Q=0.25$

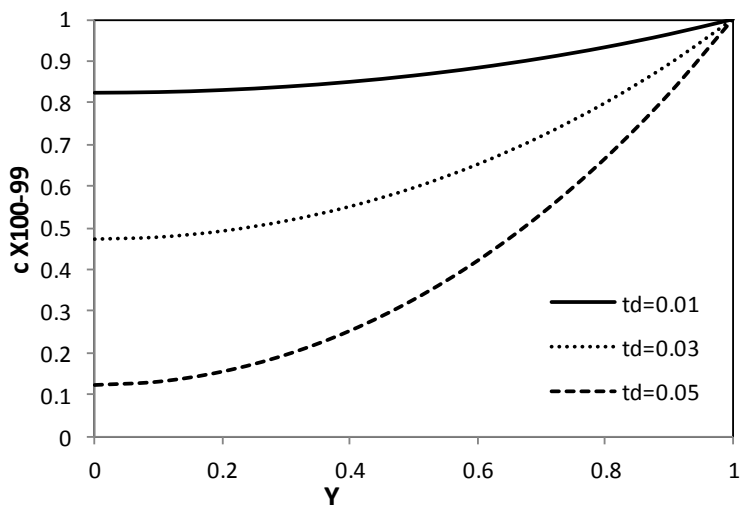

Fig.3. Graph of concentration distribution against the normalized distance for different values $t_{d}$ taking $\mathrm{M}=0.5, \mathrm{~N}=0.3$ and $\mathrm{Q}=0.25$

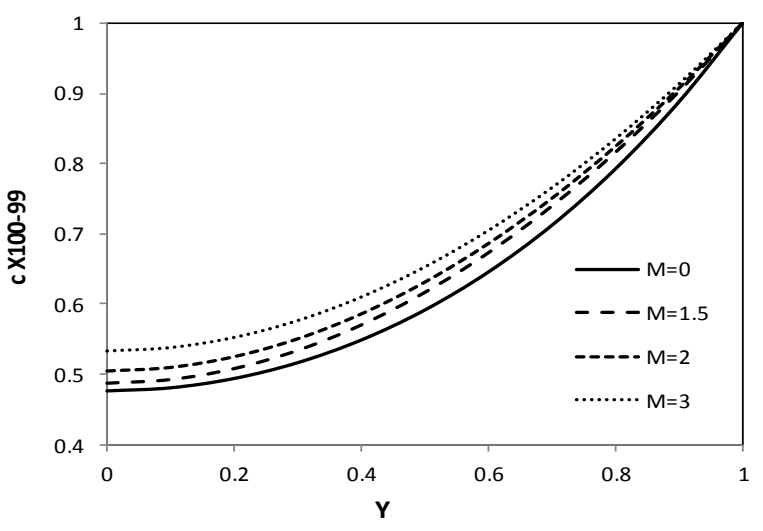

Fig.4. Graph of concentration distribution against the normalized distance for different values $t_{d}$ taking $M=0.5$, $\mathrm{N}=\mathbf{0 . 3}$ and $\mathrm{Q}=\mathbf{0 . 2 5}$

\section{ACKNOWLEDGMENT}

The authors would like to record their sincere thanks to the referee for their helpful suggestions which contribute in improving the work.

\section{REFERENCES}

[1] Vajravelu K, Hadjinicolaou A (1993) Heat transfer in a viscous fluid over a stretching sheet with viscous dissipation and internal heat generation. Int. Comm. Heat Mass Transfer 20: 417-430

[2] Mendez F, Trevino C(2000) The conjugate conduction-natural convection heat transfer along a thin vertical plate with non-uniform internal heat generation. Int. J. Heat Mass Transfer 43: 27392748

[3] Sharma BR, Singh RN, Gogoi RK(2011) Effect of a strong transverse magnetic field on separation of species of a binary fluid mixture in generalisedCouette flow. Int. J. Applied Engineering Research 6(19): 2223-2235

[4] Sharma BR, Singh RN (2007) Soret effect in natural convection between heated vertical plates in a horizontal magnetic field. J ultra SciPhySci 19 (1)M:97-106 
[5] Osterle JF, Young FJ (1961) Natural convection between heated vertical plates in a horizontal magnetic field. J. Fluid Mech. 11(4):512-518

[6] Srivastava AC ( 1992) Mass Diffusion in a Binary Mixture of Viscous Fluids. ProclNatlAcadSci 69 (A2):103-117

[7] Sharma BR, HazarikaGC(2002) Baro- diffusion and Thermal - diffusion in a Binary Mixture. Nepali Math Sci Report 20(1-2):65-73

[8] Sharma BR (2003) Baro - diffusion and Thermal diffusion in a Binary Mixture of Electrically Conducting Incompressible Fluids due to Rotation of a Circular Cylinder. Bull Pure ApplSci 22( E1):79-87

[9] Sharma BR(2004) Pressure- diffusion and Soret Effect in a Binary Fluid mixture. Bull Pure ApplSci 23(E1):165-173

[10] Sharma BR, Shah NA (1996) Baro- diffusion and Thermal - diffusion in a Binary Mixture. J Math PhySci 30(4):169-179

[11] Sharma BR,Gogoi RK (2000) The Effect Curvature of a Curved Annulus on Seperation of a Binary Mixture. In: Proceeding of the $46^{\text {th }}$ annual technical session, Assam science Society, pp 81-89

[12] Sharma BR, Singh RN (2004) Soret Effect in Generalized MHD Couette Flow of a Binary Mixture. Bull Cal Math Soc 96(5):367-374

[13] Sharma BR, Singh RN (2003) The Effect of Inclination of a Channel on Seperation of a Binary Mixture of Viscous Incompressible Thermally and Electrically conducting fluids. Proc ISTAM 48:36-43

[14] Sharma BR, Shah NA(2002) the effect of pressure gradient and teperature gradient on seperation of a binary mixture of incompressible viscous fluids filling the space outside a uniformly rotating cylinder. In: Proceeding of the $47^{\text {th }}$ annual technical session,vol. 3. Assam Science Society,pp 72-81

[15] Srivastava AC (1985) Barodiffusion in a binary mixture near a stagnation point. Nepali Math Sci Report 10(2):53-62
[16] SrivastavaAC(1999)Separation of a binary mixture of viscous fluids by thermal diffusion near a stagnation point. Ganit 50(2):129-134

[17] Srivastava AC (1999) Historical development of fluid dynamicsand mass diffusion in a binary mixture of viscous fluids. Math Stud 68( 1-4):73-85

[18] Srivastava AC ( 1991) seperation of binary mixture in a viscous fluid due to rotating heated sphere Bull GUMA 6:63-72

[19] SrivastavaPK(2003) A study of flow of two component fluids, its heat transfer.Ph.D. Thesis, University of Lucknow, Lucknow, India

[20] Srivastava AC (1979) Barodiffusion in a binary mixture confined between two disk. Math Forum 2 (2): 16-21

[21] Sharma BR, Hazarika GC, Singh RN (2006) Influence of magnetic field on seperation of a binary mixture in free convection flow considering Soret effect. J natlAcad Math 20:1-20

[22] Sharma BR, Singh RN (2007) Soret effect in natural convection between heated vertical plates in a horizontal magnetic field. J ultra SciPhySci 19 (1)M:97-106

[23] Sharma BR, Singh RN ( 2008) Baro - diffussion and thermal - diffusionin a binary fluid mixture confined between two parallel discs in presence of an small axial magnetic field. Latin Am Appl Res $38: 313-320$

[24] Sharma BR, Singh RN ( 2009) Thermal diffusion in a binary fluid mixture confined between two concentric circular cylinders in presence of radial magnetic field. J Energy Heat Mass Transfer 31:27 $-38$

[25] Sharma BR, Singh RN ( 2009) Baro - diffussion and Thermal - diffusionin a Binary Fluid Mixture Confined in an Inclined Channel in Presence of a Weak Magnetic Field. ProcNatlAcadSci India 79 ( AIII): 273-278

[26] Sharma B R, Singh R N (2010) Separation of species of a binary fluid mixture confined between two concentric rotating circular cylinders in presence of a strong radial magnetic field.Heat Mass Transfer 